\title{
Direct-acting antivirals and risk of hepatocellular carcinoma: from genetic signature to metabolic risk factors
}

\author{
Stefano Gitto', Erica Villa² \\ 'Department of Experimental and Clinical Medicine, University of Florence, Florence 50121, Italy. \\ ${ }^{2}$ Department of Medical Specialties, Gastroenterology Unit, University of Modena \& Reggio Emilia, Modena 41124, Italy. \\ Correspondence to: Prof. Erica Villa, Department of Medical Specialties, Gastroenterology Unit, University of Modena \& Reggio \\ Emilia, Via del Pozzo 71, Modena 41124, Italy. E-mail: erica.villa@unimore.it
}

How to cite this article: Gitto S, Villa E. Direct-acting antivirals and risk of hepatocellular carcinoma: from genetic signature to metabolic risk factors. Hepatoma Res 2020;6:22. http://dx.doi.org/10.20517/2394-5079.2019.50

Received: 17 Dec 2019 First Decision: 5 Mar 2020 Revised: 23 Mar 2020 Accepted: 2 Apr 2020 Published: 11 May 2020

Science Editor: Guang-Wen Cao Copy Editor: Jing-Wen Zhang Production Editor: Tian Zhang

\begin{abstract}
Hepatocellular carcinoma (HCC) is the fifth most common malignancy and the second leading cause of cancerrelated death. Hepatitis $\mathrm{C}$ virus and mainly hepatitis $\mathrm{C}$ virus-related cirrhosis is the chief risk factor for HCC. Many direct-acting antivirals are available for the eradication of hepatitis $C$ virus with remarkable results in terms of virological response and with optimal safety profile. Notably, some authors have suggested that viral eradication due to these new drugs might favor both occurrence and recurrence of HCC. The exact biological mechanisms of carcinogenesis in this specific setting have not been well identified, but it has been suggested that adjustments in immune surveillance and increase in vascular endothelial growth factor expression could have a chief role. Remarkably, after publication of many large studies and meta-analyses, we can affirm that there is no increased risk on a population basis. Nonetheless, on an individual basis, sustained virological response due to direct-acting antivirals may facilitate HCC onset in some specific subgroups of patients. Among them, we could point out patients with activated neoangiogenesis but also subjects with particularly severe metabolic imbalance.
\end{abstract}

Keywords: Direct-acting antivirals, hepatocellular carcinoma, carcinogenesis

\section{INTRODUCTION}

Hepatocellular carcinoma (HCC) represents about $5.6 \%$ of cancers worldwide ${ }^{[1]}$. It is the fifth most common malignancy and its incidence has grown in the last two decades ${ }^{[2]}$. Notably, HCC has a strong impact on patients' outcome, being the second leading cause of cancer-related death ${ }^{[3]}$. Hepatitis $\mathrm{C}$ virus $(\mathrm{HCV})$ and particularly HCV-related cirrhosis, is the main risk factor for $\mathrm{HCC}^{[4]}$.

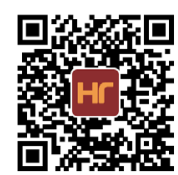


HCV leads to chronic hepatitis C and cirrhosis in about $20 \%$ of subjects within 20 years of infection ${ }^{[5]}$ and HCC occurs in around $3.5 \%$ of cases per year ${ }^{[6]}$.

For many years, interferon (IFN)-based protocols have represented the cornerstone of antiviral therapy ${ }^{[7]}$. Achievement of sustained virological response (SVR) with IFN is infrequent (especially in cirrhotics), and the drug leads to low compliance and high toxicity. However, viral clearance due to IFN is associated with decreased incidence of HCC and overall reduction of other liver-related events ${ }^{[8]}$.

In the last few years, new antiviral molecules with a direct action against the virus have been developed ${ }^{[9]}$. Today, many direct-acting antivirals (DAAs) are available with amazing results in terms of SVR and safety ${ }^{[10]}$.

A few years after the introduction of DAAa, two noteworthy and innovative studies reported first that SVR due to DAA might increase the risk of both HCC occurrence and recurrence ${ }^{[11,12]}$.

Later, other authors suggested the unexpected high incidence of HCC after DAA treatment, describing the onset of extraordinarily aggressive tumors a few weeks after the end of antiviral therapy ${ }^{[13]}$.

Following the publication of these studies, many other groups reported the increased occurrence of HCC after DAA use ${ }^{[14]}$, whereas others rebutted this notion ${ }^{[15-17]}$.

The exact biological mechanisms by which oral DAAs might enhance the risk of HCC are largely unknown ${ }^{[18]}$. Modifications in immune surveillance may potentiate the risk of HCC by accelerating the process of regeneration. Moreover, DAAs seem to increase levels of vascular endothelial growth factor (VEGF) and to modify the balance between pro- and anti-inflammatory reactions ${ }^{[19]}$.

The aim of the present review was to analyze the main evidence about the relationship between DAA and HCC onset, privileging meta-analyses and original real-life controlled trials. Moreover, we tried to identify high-risk subpopulations that can benefit from personalized post-eradication follow-up. We analyzed the state-of-the-art about this debated matter offering a critical point of view and underlining potential future perspectives.

\section{HCC RECURRENCE AFTER SVR DUE TO DAA THERAPY}

In the last years, there has been particular concern about the rates of HCC recurrence following DAA treatment $^{[11,12,20]}$.

Notably, many studies about HCC recurrence are single-arm and retrospective cohort studies. Moreover, they show relevant heterogeneity in the following patterns: tumor burden, HCC treatments leading to whole response, and follow-up periods ${ }^{[21]}$. Indeed, it is questionable whether HCC recurrence might be part of the natural history of patients with advanced liver disease or whether DAAs can have a chief role in cancer pathogenesis. Fortunately, in the last 2 years, some meta-analyses have been proposed, and these are the ideal instruments to obtain solid answers.

In 2017, Waziry et al. ${ }^{[22]}$ compared, using systematic review, meta-analysis, and meta-regression approaches, 15 studies on HCC recurrence after SVR. Seven studies included patients treated with IFN-based therapy and 10 comprising patients cured with DAA. The incidence of HCC recurrence was 9.21/100 per year and 12.16/100 per year in IFN and DAA subgroups, respectively. Through a meta-regression analysis and an adjustment for age and follow-up length, the authors demonstrated that patients in the DAA group did not show higher HCC recurrence in comparison with those treated with IFN. 
Sometime later, Saraiya et al. ${ }^{[21]}$ conducted a further meta-analysis examining data from 1820 patients with SVR due to DAA treatment. A vast part of the studies considered involved less than 100 patients with consequent inexact estimates of HCC recurrence. Notably, many but not all patients were treated for HCC with radical therapy (surgical resection or ablation). In fact, a substantial percentage of subjects (25\%-50\%) received non-curative therapies such as transarterial chemoembolization, showing by definition a high risk of recurrence regardless of antiviral therapy.

The authors correctly underlined other methodological differences among the studies analyzed. The extent of follow-up to evaluate HCC recurrence varied among the studies (from 3 to 36 months). Furthermore, some groups defined HCC recurrence from date of cancer treatment, while others assessed recurrence from start of DAAs.

According to reported data, the HCC recurrence rate was $21.9 \%$ (with high statistical heterogeneity). Nine studies compared HCC recurrence in DAA-treated $(n=947)$, IFN-treated $(n=210)$ and/or untreated $(n=$ 641) patients. The authors demonstrated that DAA-treated patients displayed lower pooled recurrence risk than did untreated patients and similar rate in comparison with subjects treated with IFN-based protocols.

In the same year (2018), another meta-analysis was published ${ }^{[23]}$. The authors selected studies better with respect to the previous meta-analysis, since they restricted patient enrollment to those with HCC previously treated with a curative approach (surgical resection or percutaneous ablation). The authors subdivided uncontrolled and controlled studies. The pooled proportion of recurrent HCC in uncontrolled cohorts was $16.7 \%$ (with evidence of substantial statistical heterogeneity) with follow-up that highly varied (from 12 to 280 weeks). Considering controlled cohorts, the pooled proportion was $20.1 \%$ (again with substantial statistical heterogeneity) with a follow-up range that was up to 416 weeks. Remarkably, the authors themselves reported that the global quality of the evidence was low for each of the outcomes considered, suggesting the need for further studies with longer follow-up periods.

Recently, Rutledge et al. ${ }^{[24]}$ made strong suggestions about the complex matter of HCC recurrence after DAA therapy. The meta-analysis conducted by these authors is not only the widest among the available studies but is also particularly solid from a methodological point of view. In fact, the authors included only patients with previous curative cancer therapy and excluded studies with less than one year of follow-up. Notably, this rigorous approach allowed avoiding any rise in early HCC recurrence that could definitely be associated with the presence of small subclinical HCC at the beginning of antiviral therapy. The authors analyzed and compared 57 studies, which involved 16 IFN-treated, 33 DAA-treated and 8 untreated patients. DAA-treated patients who achieved SVR displayed a recurrence rate of 18.17/100 per year. Patients with SVR due to IFN therapy showed a similar recurrence rate (11.01/100 per year). The rate of HCC recurrence was comparable between untreated patients (25.69/100 per year) and IFN-treated patients without virological response (16.89/100 per year); subjects not responding to DAA showed a higher rate of recurrence, but the small number of patients made the value not statistically significant (44.16/100 per year).

Notably, the authors performed a subanalysis adjusting data according to HCC consolidated risk factors such as age and cirrhosis. They demonstrated that the DAA-treated group showed a lower rate of recurrent HCC (although without statistical significance).

Since overall mortality represents the most important clinical outcome, the recent findings of Singal et al. ${ }^{[25]}$ should be pointed out. The authors demonstrated with a multicenter cohort study $(n=797)$, that patients who achieved both complete response to any HCC treatment (resection, local ablation, transarterial chemoor radioembolization, or radiation therapy) and SVR due to DAAs, showed a significant reduction of mortality risk in comparison with non-responders and untreated subjects. 


\section{HCC OCCURRENCE AFTER SVR ASSOCIATED WITH DAA THERAPY}

Chronic viral hepatitis is the most important predisposing factor for the occurrence of $\mathrm{HCC}^{[4]}$. Indeed, viral eradication is a main goal to decrease the incidence of HCC and, more in general, to lessen liver-related mortality ${ }^{[3,8]}$.

Considering the IFN era, Van der Meer et al. ${ }^{[26]}$, compared long-term outcomes of IFN monotherapy, IFN + ribavirin and PEG IFN + ribavirin, in 5 Liver Units. Recruited patients displayed advanced fibrosis and were followed up for a mean period of 8.4 years. Ten-year cumulative HCC incidence rate was $5.1 \%$ for the SVR subgroup and $21.8 \%$ for non-responders.

Ogawa et al. ${ }^{[27]}$ conducted a prospective national multicenter study on the effect of PEG IFN + ribavirin treatment on chronic HCV infection, focusing on HCC occurrence. The authors enrolled 1013 patients and demonstrated that 5-year cumulative incidence of HCC was $1.7 \%$ in the SVR and $7.6 \%$ in the non-SVR group with a weakening of this effect considering only cirrhotic subjects (18.9\% vs. 39.4\%).

Morgan et al. ${ }^{[28]}$ carried out a meta-analysis including 30 observational studies, with 31,528 patients enrolled. The authors confirmed that patients with SVR due to IFN-based protocol showed a lower HCC occurrence with respect to the others: 0.33 per person-year in SVR and 1.67 in non-responders.

Regarding the role of DAA therapy in favoring HCC occurrence, Conti et al. ${ }^{[11]}$ published in 2016 a regional multicenter study of 344 consecutive cirrhotic patients treated with DAAs, including 285 subjects who did not show previous HCC. The authors reported that after the end of DAA treatment (follow-up of 24 weeks), 9 patients $(3.16 \%)$ developed HCC in a higher percentage than IFN responders. The key point of discussion should be about the main clinical patterns of enrolled patients who displayed more advanced liver disease in comparison with the classical IFN cohort. Additionally, enrolled patients were screened for HCC exclusively by ultrasound with a certain probability of small undiagnosed HCC nodules at the beginning of antiviral therapy.

Some of the previously cited meta-analyses examined the whole risk of HCC occurrence after DAAs.

Waziry et al. ${ }^{[22]}$, comparing IFN regimens and DAAs, demonstrated that HCC occurrence following SVR was $1.14 / 100$ per year for patients treated with IFN and 2.96/100 per year in subjects receiving DAAs. Notably, the authors showed that occurrence rate decreased with longer follow-up and was lower in younger subjects. Finally, the authors proposed a meta-regression after adjusting data for follow-up and age, demonstrating that SVR due to DAA was not related to significantly more HCC occurrence.

Singh et al. ${ }^{[23]}$ analyzed a total of 39,145 patients treated with DAA (data from uncontrolled studies). Among them, 542 developed de novo HCC after achievement of SVR. Incident HCC showed an overall occurrence of $1.5 \%$ with substantial statistical heterogeneity among the studies included. The authors examined the HCC occurrence rate associated with DAA considering only controlled cohorts. Among five studies, the proportion of HCC occurrence was 3.3\%, again with considerable statistical heterogeneity. Therefore, authors concluded that data derived from their study should be considered non-conclusive regarding the possible relationship between DAAs and increased risk of HCC occurrence.

A recently published meta-analysis ${ }^{[24]}$ examined 81 studies of HCC occurrence after DAA. In particular, the authors compared the following subgroups of studies: 31 involving 71,443 IFN-treated subjects, 44 comprising 91,249 of DAA-treated patients and 6 on 9944 untreated subjects.

The authors reported that, considering only patients with SVR due to DAA and not all the DAA population, HCC occurrence rate was 3.57 per 100 per year vs. 0.70 per 100 per year in the IFN-treated SVR group 
(without statistical significance). Afterwards, the authors carried out an additional smaller meta-analysis evaluating only studies with multivariate-adjusted hazard ratios (for gender, baseline cirrhosis and age). With this approach, these authors did not find augmented hazard of HCC occurrence in subjects treated with DAA compared to the IFN-treated subgroup. Notably, the authors also sub-analyzed data according to follow-up length. Considering occurrence rates in the second year after treatment with DAAs, similar incidence rates were found ( 0.88 per 100 per year in the DAA group and 0.55 in the IFN group). Finally, the authors conducted a sub-analysis excluding any cases of HCC occurrence within six months after end oftreatment, where they found an incidence rate of 1.12 per 100 per year for the DAA group and 3.01 for the IFN group (again without statistical significance).

\section{HIGH RISK PATIENTS: POTENTIAL ROLE OF GENETIC SIGNATURE}

The data outlined in the previous paragraphs underlined the concepts that on the whole, the risk of developing HCC after DAAs is small and DAAs represent an extraordinary option for curing hepatitis C. There is already consistent evidence that on a population basis, DAAs improve survival and decrease longterm complications such as decompensation. On an individual basis, however, DAAs might constitute a stimulus associated with higher risk of developing HCCs. In a series of patients with liver cirrhosis enrolled in a prospective study of HCC development, liver tissue of patients who subsequently developed HCC were already primed towards neoangiogenesis well before DAA treatment and ensuing HCC development ${ }^{[2]}$. Expression levelsof angiopoietin-2, evaluated by immunohistochemistry, differed significantly in tumor tissue among patients with recurrent, de novo, and non-recurrent HCC after DAAs $(P<0.0001$, KruskalWallis test). Likewise, hepatic stiffness, spleen size, variceal size, and hepatic venous pressure gradient were related to HCC risk but only angiopoietin-2 was independently related to both risk of HCC occurrence and recurrence. Our hypothesis was that the events occurring during the progression of chronic liver disease, with occurrence of increasing portal hypertension, could be critical in activating angiopoietin-2 expression in splanchnic vascular bed hyperplasia ${ }^{[29]}$. Of further interest, HCC developing after DAAs was often biologically aggressive, as indicated in a large proportion of them by the presence of five-gene neoangiogenic signature identified as a marker of rapidly growing and clinically aggressive $\mathrm{HCC}^{[30]}$.

The intriguing relationship between severe portal hypertension and DAA-mediated carcinogenesis could already be glimpsed in a few previously published papers. Conti et al. ${ }^{[1]}$ identified more severe liver fibrosis as independently associated with risk of HCC recurrence. Ravaioli et al.$^{[31]}$ recently showdc that the decrease in spleen stiffness in patients who developed HCC after DAAs was significantly less than in patients who did not develop HCC. Similar results were obtained by Ioannou et al. ${ }^{[32]}$ who showed that when FIB-4 remains higher than 3.25 after SVR, the risk of HCC is so consistent to merit HCC surveillance. This highlights that severe portal hypertension is a critical predisposing factor for HCC.

\section{IMPACT OF METABOLIC RISK FACTORS ON HCC RISK}

Both diabetes and obesity represent consolidated risk factors for HCC exerting a strong carcinogenic effect directly by disrupting the insulin-IGF pathway or indirectly by promoting steatosis ${ }^{[33]}$.

In a large retrospective cohort study, El-Serag et al. ${ }^{[34]}$ showed that SVR due to effective IFN-based treatment led to a noteworthy drop in HCC risk. Nevertheless, the authors reported that the annual risk of HCC among responding patients was not insignificant (overall $0.33 \%$ ). Interestingly, residual HCC risk was associated not only with advanced stage of fibrosis and age but also with diabetes and HCV genotype 3. Relative to this point, other authors also suggested that HCV genotype 3 was correlated not only with higher rates of liver steatosis (being the more "metabolic" genotype) but also with greater HCC risk in comparison with the other genotypes ${ }^{[35,36]}$. These data indicate that metabolic disorders might have a significant role also in determining HCC occurrence after DAA therapy. 
Table 1. Available meta-analyses analyzing the possible association between DAA therapy and HCC

\begin{tabular}{|c|c|c|c|c|c|c|}
\hline Ref. & Year & Country & $\mathbf{N}$ of studies & $\mathbf{N}$ of patients & Increased risk of occurrence & Increased risk of recurrence \\
\hline Waziry et al. ${ }^{[22]}$ & 2017 & Australia & 41 & 13,875 & DAA comparable to IFN & DAA comparable to IFN \\
\hline Saraiya et al. ${ }^{[21]}$ & 2018 & USA & 24 & 1820 & / & DAA comparable to IFN \\
\hline Singh et al. ${ }^{[23]}$ & 2018 & USA & 44 & 39,145 & no conclusive data & no conclusive data \\
\hline Rutledge et $a$ I. $^{[24]}$ & 2019 & USA & 138 & 177,512 & DAA comparable to IFN & DAA comparable to IFN \\
\hline
\end{tabular}

DAA: direct-acting antiviral; HCC: hepatocellular carcinoma; IFN: interferon

Regarding the possible relationship between metabolic disorders and risk of HCC in the specific setting of DAA therapy, Nahon et al ${ }^{[37]}$ reported interesting results. These authors retrospectively analyzed data from 1270 cirrhotic patients comparing the incidence of HCC in patients treated with IFN-based protocols or DAA therapy. First of all, authors demonstrated that patients treated with DAA were older and had higher rates of diabetes, suggesting that these patterns might explain the reported higher percentages of HCC occurrence in this subgroup of patients in comparison with the IFN group. However, the authors conducted a multivariate analysis, demonstrating that DAA therapy itself did not emerge as a predictor of HCC occurrence. Other factors such as increased age, past excessive alcohol consumption, virological parameters [genotype 1 (in contrast with previously reported data)], decreased platelet count and increased gamma-glutamyl transferase (GGT) levels were independently associated with post-treatment HCC onset. Concerning the metabolic issue, neither diabetes nor obesity was directly associated with HCC occurrence, but it is well known that GGT increase is a sign of altered metabolism ${ }^{[38]}$ or higher inflammatory activity ${ }^{[39]}$. In fact, elevated GGT activity is coupled with traditional cardiovascular risk factors and particularly with metabolic syndrome ${ }^{[38]}$.

\section{CONCLUSIONS AND FUTURE PERSPECTIVES}

DAAs have constituted an extraordinary breakthrough in the therapy of hepatitis C. With an 8- to 12-week course of treatment, SVR occurs in more than $95 \%-99 \%$ of patients, depending on degree of liver fibrosis. These results were beyond any reasonable expectation until a few years ago. It is therefore quite conceivable that the report of an increased occurrence or recurrence of HCC during or shortly after stopping DAAs initiated a series of reactions of concern. With more data accumulating, it is now clear that there is no such risk on a population basis. In fact, as summarized in Table 1, the available high-quality meta-analyses categorically demonstrated that the risk of both HCC recurrence and occurrence after SVR is comparable between patients treated with "old" IFN-based protocols and new DAAs. As recently stated by the American Association for the Study of Liver Diseases ${ }^{[40]}$, SVR due to DAAs determine a decrease in all-cause mortality, cirrhosis, hepatic decompensation and HCC, an improvement in extra-hepatic manifestations of HCV, and an amelioration of both productivity and quality of life. Summarizing, we must treat with DAA all patients without history of HCC and all subjects with complete response to HCC treatment. Notably, as strongly demonstrated in a recently published meta-analysis ${ }^{[4]}$, patients with HCC show, for unknown reasons, lower pooled SVR rate in comparison with subjects without cancer ( $88.2 \%$ vs. 92.4\%).

Although DAAs represent a great therapeutic option, it is possible that some subgroups of subjects may be at increased risk of HCC onset after DAA treatment. In fact, on an individual basis, administration of DAAs might be associated with facilitated HCC development in subjects who already have a predisposing hepatic condition, namely activated neoangiogenesis, as shown by increased levels of angiopoietin-2 in cirrhotic tissue ${ }^{[29]}$. In these patients, a careful evaluation of risk-benefit of DAA treatment should be done, also in view of the aggressive features of DAA-associated $\mathrm{HCC}^{[29]}$. Finally, in the long-term management of HCVpositive patients, it has to be considered that subjects with severe metabolic impairment seem to maintain a significant risk of HCC despite antiviral treatment and viral eradication ${ }^{[37]}$.

Concerning the possible identification of subpopulations at higher risk of HCC, some authors have analyzed the change in inflammatory balance due to DAAs as a determinant of cancer development ${ }^{[1,42]}$. 
In particular, Villani et al. ${ }^{[19]}$ studied 103 patients treated with DAAs. Changes in levels of VEGF, epidermal growth factor, and several interleukins were evaluated. Interestingly, the authors demonstrated that VEGF increased 4-fold from baseline to week 4 of treatment. VEGF level continued to increase until the end of treatment and returned to the pre-DAA level afterwards. Of note, VEGF induces angiopoietin-2 expression and can therefore promote DAA-induced carcinogenesis as described in ${ }^{[2]]}$. On the other hand, interleukin-10 and tumor necrosis factor-alpha significantly decreased with HCV clearance. The authors of the cited study concluded that DAAs can alter the balance between inflammatory and anti-inflammatory processes, affecting antitumor surveillance of the host and favoring HCC onset after DAA therapy.

In conclusion, available data indicate that DAAs represent a great option for both patients without cancer and subjects with effectively treated HCC. Further studies may confirm whether or not some high-risk subgroups exist, which deserve a personalized surveillance approach.

\section{DECLARATIONS}

\section{Authors' contributions}

Conceived and designed the study and wrote the article together: Gitto S, Villa E

\section{Availability of data and materials}

Not applicable.

\section{Financial support and sponsorship}

None.

\section{Conflicts of interest}

Both authors declared that there are no conflicts of interest.

\section{Ethical approval and consent to participate}

Not applicable.

\section{Consent for publication}

Not applicable.

\section{Copyright}

(c) The Author(s) 2020.

\section{REFERENCES}

1. Ferlay J, Soerjomataram I, Dikshit R, Eser S, Mathers C, et al. Cancer incidence and mortality worldwide: sources, methods and major patterns in GLOBOCAN 2012. Int J Cancer 2015;136:E359-86.

2. Beste LA, Leipertz SL, Green PK, Dominitz JA, Ross D, et al. Trends in burden of cirrhosis and hepatocellular carcinoma by underlying liver disease in US veterans, 2001-2013. Gastroenterology 2015;149:1471-82.

3. D'Ambrosio R, Della Corte C, Colombo M. Hepatocellular carcinoma in patients with a sustained response to anti-hepatitis C therapy. Int J Mol Sci 2015;16:19698-712.

4. Fattovich G, Stroffolini T, Zagni I, Donato F. Hepatocellular carcinoma in cirrhosis: incidence and risk factors. Gastroenterology 2004;127:S35-50.

5. Thein HH, Yi Q, Dore GJ, Krahn MD. Estimation of stage-specific fibrosis progression rates in chronic hepatitis C virus infection: a meta-analysis and meta-regression. Hepatology 2008;48:418-31.

6. Sangiovanni A, Del Ninno E, Fasani P, De Fazio C, Ronchi G, et al. Increased survival of cirrhotic patients with a hepatocellular carcinoma detected during surveillance. Gastroenterology 2004;126:1005-14.

7. Yano H, Iemura A, Haramaki M, Ogasawara S, Takayama A, et al. Interferon alfa receptor expression and growth inhibition by interferon alfa in human liver cancer cell lines. Hepatology 1999;29:1708-17.

8. Simmons B, Saleem J, Heath K, Cooke GS, Hill A. Long-term treatment outcomes of patients infected with hepatitis C virus: a systematic review and meta-analysis of the survival benefit of achieving a sustained virological response. Clin Infect Dis 2015;61:730-40.

9. Conteduca V, Sansonno D, Russi S, Pavone F, Dammacco F. Therapy of chronic hepatitis C virus infection in the era of direct-acting 
and host-targeting antiviral agents. J Infect 2014;68:1-20.

10. Sandmann L, Schulte B, Manns MP, Maasoumy B. Treatment of chronic hepatitis C: efficacy, side effects and complications. Visc Med 2019;35:161-70

11. Conti F, Buonfiglioli F, Scuteri A, Crespi C, Bolondi L, et al. Early occurrence and recurrence of hepatocellular carcinoma in HCVrelated cirrhosis treated with direct-acting antivirals. J Hepatol 2016;65:727-33.

12. Reig M, Mariño Z, Perelló C, Iñarrairaegui M, Ribeiro A, et al. Unexpected high rate of early tumor recurrence in patients with $\mathrm{HCV}$ related HCC undergoing interferon free therapy. J Hepatol 2016;65:719-26.

13. Kozbial K, Moser S, Schwarzer R, Laferl H, Al-Zoairy R, et al. Unexpected high incidence of hepatocellular carcinoma in cirrhotic patients with sustained virologic response following interferon-free direct-acting antiviral treatment. J Hepatol 2016;65:856-8.

14. Rinaldi L, Di Francia R, Coppola N, Guerrera B, Imparato M, et al. Hepatocellular carcinoma in HCV cirrhosis after viral clearance with direct acting antiviral therapy: preliminary evidence and possible meanings. WCRJ 2016;3:e748.

15. Ioannou GN, Green PK, Berry K. HCV eradication induced by direct-acting antiviral agents reduces the risk of hepatocellular carcinoma. J Hepatol 2017.

16. ANRS collaborative study group on hepatocellular carcinoma. Lack of evidence of an effect of direct-acting antivirals on the recurrence of hepatocellular carcinoma: data from three ANRS cohorts. J Hepatol 2016;65:734-40.

17. Ikeda K, Kawamura Y, Kobayashi M, Kominami Y, Fujiyama S, et al. Direct-acting antivirals decreased tumor recurrence after initial treatment of hepatitis C virus-related hepatocellular carcinoma. Dig Dis Sci 2017;62:2932-42.

18. Grandhe S, Frenette CT. Occurrence and recurrence of hepatocellular carcinoma after successful direct-acting antiviral therapy for patients with chronic hepatitis C virus infection. Gastroenterol Hepatol 2017;13:421-5.

19. Villani R, Facciorusso A, Bellanti F, Tamborra R, Piscazzi A, et al. DAAs rapidly reduce inflammation but increase serum VEGF Level: a rationale for tumor risk during anti-HCV treatment. PLoS One 2016;11:e167934.

20. El Kassas M, Funk AL, Salaheldin M, Shimakawa Y, Eltabbakh M, et al. Increased recurrence rates of hepatocellular carcinoma after DAA therapy in a hepatitis C infected Egyptian cohort: a comparative analysis. J Viral Hepat 2018;25:623-30.

21. Saraiya N, Yopp AC, Rich NE, Odewole M, Parikh ND, et al. Systematic review with meta-analysis: recurrence of hepatocellular carcinoma following direct acting antiviral therapy. Aliment Pharmacol Ther 2018;48:127-37.

22. Waziry R, Hajarizadeh B, Grebely J, Amin J, Law M, et al. Hepatocellular carcinoma risk following direct-acting antiviral HCV therapy: a systematic review, metaanalyses, and meta-regression. J Hepatol 2017;67:1204-12.

23. Singh S, Nautiyal A, Loke YK. Oral direct-acting antivirals and the incidence or recurrence of hepatocellular carcinoma: a systematic review and meta-analysis. Frontline Gastroenterol 2018;9:262-70.

24. Rutledge SM, Zheng H, Li DK, Chung RT. No evidence for higher rates of hepatocellular carcinoma after direct-acting antivira treatment: a meta-analysis. Hepatoma Res 2019;5:31.

25. Singal AG, Rich NE, Mehta N, Branch AD, Pillai A, et al. Direct-acting antiviral therapy for hepatitis C virus infection is associated with increased survival in patients with a history of hepatocellular carcinoma. Gastroenterology 2019;157:1253-63.e2.

26. Van der Meer AJ, Veldt BJ, Feld JJ, Wedemeyer H, Dufour JF, et al. Association between sustained virological response and all-cause mortality among patients with chronic hepatitis $\mathrm{C}$ and advanced hepatic fibrosis. JAMA 2012;308:2584-93.

27. Ogawa E, Furusyo N, Kajiwara E, Takahashi K, Nomura H, et al. Efficacy of pegylated interferon alpha-2b and ribavirin treatment on the risk of hepatocellular carcinoma in patients with chronic hepatitis C: a prospective, multicenter study. J Hepatol 2013;58:495-501.

28. Morgan RL, Baack B, Smith BD, Yartel A, Pitasi M, et al. Eradication of hepatitis C virus infection and the development of hepatocellular carcinoma: a meta-analysis of observational studies. Ann Intern Med 2013;158:329-37.

29. Faillaci F, Marzi L, Critelli R, Milosa F, Schepis F, et al. Liver angiopoietin-2 is a key predictor of de novo or recurrent hepatocellular cancer after hepatitis $\mathrm{C}$ virus direct-acting antivirals. Hepatology 2018;68:1010-24.

30. Villa E, Critelli R, Lei B, Marzocchi G, Cammà C, et al. Neoangiogenesis-related genes are hallmarks of fast-growing hepatocellular carcinomas and worst survival. Results from a prospective study. Gut 2016;65:861-9.

31. Ravaioli F, Conti F, Brillanti S, Andreone P, Mazzella G, et al. Hepatocellular carcinoma risk assessment by the measurement of liver stiffness variations in HCV cirrhotics treated with direct acting antivirals. Dig Liver Dis 2018;50:573-9.

32. Ioannou GN, Beste LA, Green PK, Singal AG, Tapper EB, et al. Increased risk for hepatocellular carcinoma persists up to 10 years after HCV eradication in patients with baseline cirrhosis or high FIB-4 scores. Gastroenterology 2019;157:1264-78.

33. El-Serag HB, Kanwal F. Obesity and hepatocellular carcinoma: hype and reality. Hepatology 2014;60:779-81

34. El-Serag HB, Kanwal F, Richardson P, Kramer J. Risk of hepatocellular carcinoma after sustained virological response in Veterans with hepatitis C virus infection. Hepatology 2016;64:130-7.

35. Kanwal F, Kramer JR, Ilyas J, Duan Z, El-Serag HB. HCV genotype 3 is associated with an increased risk of cirrhosis and hepatocellular cancer in a national sample of U.S. Veterans with HCV. Hepatology 2014;60:98-105.

36. Kanwal F, White DL, Jiao L, Tavakoli-Tabasi S, Sansgiry S, et al. Genetic variants in interleukin-28B are associated with diabetes and diabetes-related complications in patients with chronic hepatitis C virus infection. Dig Dis Sci 2015;60:2030-7.

37. Nahon P, Layese R, Bourcier V, Cagnot C, Marcellin P, et al. Incidence of hepatocellular carcinoma after direct antiviral therapy for HCV in patients with cirrhosis included in surveillance programs. Gastroenterology 2018;155:1436-50.

38. Ndrepepa G, Colleran R, Kastrati A. Gamma-glutamyl transferase and the risk of atherosclerosis and coronary heart disease. Clin Chim Acta 2018:476:130-8.

39. Taliani G, Badolato MC, Nigro G, Biasin M, Boddi V, et al. Serum concentration of gammaGT is a surrogate marker of hepatic TNFalpha mRNA expression in chronic hepatitis C. Clin Immunol 2002;105:279-85.

40. Ghany MG, Morgan TR; AASLD-IDSA Hepatitis C Guidance Panel. Hepatitis C guidance 2019 update: american association for the study of liver diseases-infectious diseases society of america recommendations for testing, managing, and treating hepatitis $\mathrm{C}$ virus infection. Hepatology 2020;71:686-721. 
41. He S, Lockart I, Alavi M, Danta M, Hajarizadeh B, et al. Systematic review with meta-analysis: effectiveness of direct-acting antiviral treatment for hepatitis C in patients with hepatocellular carcinoma. Aliment Pharmacol Ther 2020;51:34-52.

42. Casadei Gardini A, Foschi FG, Conti F, Petracci E, Vukotic R, et al. Immune inflammation indicators and ALBI score to predict liver cancer in HCV-patients treated with direct-acting antivirals. Dig Liver Dis 2019;51:681-8. 